\title{
Sexagem histológica e desempenho de Oreochromis niloticus testando diâmetros de ração de acordo com o aparato bucal
}

\author{
[Histological gender diagnosis and performance of Oreochromis niloticus testing diameters of \\ crumble diet according to oral apparatus] \\ L.S.O. Nakaghi ${ }^{1}$, M.C.F. Paes ${ }^{1}$, L.C. Makino ${ }^{1}$, T.C.R. Dias- Koberstein ${ }^{1}$, E.B. Malheiros ${ }^{2}$ \\ ${ }^{1}$ Centro de Aquicultura - UNESP \\ Via de Acesso Prof. Paulo Donato Castellane s/n \\ 14884-900 - Jaboticabal, SP \\ ${ }^{2}$ Faculdade de Ciências Agrárias e Veterinárias - UNESP - Jaboticabal, SP
}

\begin{abstract}
RESUMO
Estudou-se o tamanho da boca de larvas de tilápia-do-nilo e testou-se o efeito de diferentes granulometrias da ração sobre o ganho de peso, comprimento e sobrevivência das larvas aos 30 e 60 dias de arraçoamento. Avaliou-se também o método de sexagem por meio de microscopia de luz aos 35 dias de idade. A medida da boca das larvas apresentou valores médios de $918,2 \pm 152,9 \mu \mathrm{m}$ aos cinco dias de idade. De acordo com esse dado, testaram-se três granulometrias: $0,25,0,35$ e $0,50 \mathrm{~mm}$. Aos 30 e aos 60 dias de arraçoamento, $10 \%$ das larvas foram medidas, pesadas e contadas para cálculo da taxa de sobrevivência. O tamanho dos grânulos testados não afetou o desempenho das larvas de tilápia nilótica com alimentação iniciada aos cinco dias pós-eclosão. Quanto à sexagem histológica aos 35 dias de idade, as gônadas apresentaram-se, em sua maioria, indiferenciadas. Recomenda-se que essa análise deva ser realizada de acordo com o tamanho dos animais e não com a idade.
\end{abstract}

Palavras-chave: peixe, boca, gônadas, granulometria, histologia

\begin{abstract}
The mouth size of Nile tilapia larvae and the effect of different diameters of crumble fish food over weight gain, total length, and survival of larvae after 30 and 60 days of feeding were studied. The method of gender diagnosis based on light microscopy at 35 days of age was also evaluated. The larval mouth measurement presented average values of $918.2 \pm 152.9 \mu \mathrm{m}$ at five days of age. Based on this information, three granule sizes were tested: $0.25,0.35$, and $0.50 \mathrm{~mm}$. At 30 and 60 days of feeding, $10 \%$ of larvae were measured, weighed, and counted to calculate the survival rate. It was verified that the crumble size did not affect the performance of Nile tilapia larvae when feeding was initiated five days after hatching. In relation to the histological gender diagnosis at 35 days of age, most of gonads were undifferentiated. Therefore, it is recommended that this analysis should be carried out according to the size of animals instead of their age.
\end{abstract}

Keywords: fish, mouth, gonads, granule size, histology

\section{INTRODUÇÃO}

Dentre as atividades agroindustriais existentes, a piscicultura é reconhecida como uma das mais importantes, capaz de gerar grande retorno financeiro para os produtores e para as indústrias processadoras de peixes (Pinheiro et al., 2006). Entre as diversas espécies cultivadas, destaca-se a tilápia nilótica que se tornou rapidamente uma cultura popular de peixe, devido à sua rusticidade, alta taxa de crescimento, curto período de maturação, fácil manipulação e

Recebido em 7 de agosto de 2008

Aceito em 23 de abril de 2009

E-mail: laurankg@fcav.unesp.br 
também por ser receptiva à fertilização artificial (Galman e Avtalion, 1989). Apresenta ainda muitas características favoráveis para o desenvolvimento de projetos em piscicultura, pois pode ser uma importante fonte de renda e de proteína animal para o consumo humano, possuindo carne de excelente qualidade e com grande aceitação no mercado.

O gênero Oreochromis está entre os mais importantes peixes comercialmente produzidos na aquicultura mundial, com mais de 75 países produtores (Morrison et al., 2001). Porém é uma espécie que apresenta alta capacidade de reprodução, maturação precoce e baixa competição intraespecífica, sendo que estes fatores acarretam um quadro de superpopulação que prejudica o rendimento da produção. Para amenizar esses problemas, têm sido estudados vários métodos de controle da excessiva reprodução das tilápias e o principal deles é a manipulação do sexo fenotípico do peixe por meio da administração oral de esteroides sexuais para produção de machos. Portanto, a forma de alimentação da tilápia na fase larval é bastante importante, pois é durante esse período que se faz a reversão sexual adicionando hormônio masculinizante à ração, a qual é fornecida aos animais (Phelps et al., 1995; Carrasco et al., 1999; Meurer et al., 2003). A fase de diferenciação do sexo na reversão varia conforme as condições experimentais de temperatura, densidade de estocagem, quantidade de ração oferecida, tipo e dosagem do hormônio empregado, via de administração e condições fisiológicas particulares de cada espécie (Carvalho e Foresti, 1996). Alguns trabalhos de reversão sexual mostram experimentos em que o fornecimento de ração, com a presença de hormônio masculinizante, dura de 20 a 45 dias (Mainardes-Pinto et al., 2000; Toyama et al., 2000; Makino, 2005). Todavia, as análises histológicas das gônadas são realizadas posteriormente a esse período, quando os animais já atingiram um tamanho considerável.

A tilápia nilótica, ao contrário da maioria dos peixes, desenvolve-se bem no período larval alimentando-se somente com ração artificial (Santiago et al., 1987; Hayashi et al., 1999; Meurer et al., 2005).

Ao formular uma ração, além do balanço nutricional, deve-se levar em conta o seu processamento adequado, de modo que a dieta apresente propriedades que permitam seu rápido consumo e utilização pelos animais (Nutrient ..., 1993).

No processamento de rações, a granulometria mostra-se como um dos fatores mais importantes, pois ela deve ser compatível ao tamanho da boca da larva no momento de início da alimentação e, também, porque quanto mais finamente moída, mais alto é o custo de produção, devido ao maior consumo de tempo e energia (Meurer et al., 2005). Além disso, o grau de moagem pode alterar as propriedades físicas, estabilidade, durabilidade, dureza e seletividade dos peletes por parte dos animais (Booth et al., 2000), juntando-se ainda a perda de nutrientes por lixiviação na superfície do corpo d'água, aumentando a carga poluente no mesmo e naquele que recebe os efluentes (Pezzato, 1999).

Uma vez determinada a granulometria da ração, pode-se evitar o arraçoamento incorreto, prevenindo, assim, a deterioração da qualidade da água, que é de fundamental importância, além de melhorar a eficiência de reversão sexual em tilápias, devido ao consumo ideal de ração contendo hormônio necessário para tal técnica (Meurer et al., 2005).

Assim, os objetivos do presente trabalho foram avaliar o efeito de três granulometrias de ração relacionando com o desenvolvimento do aparato bucal de larvas de tilápia-do-nilo durante 60 dias de arraçoamento sobre parâmetros de desempenho e analisar as gônadas de juvenis revertidos sexualmente aos 35 dias de idade, para observar a diferenciação do sexo relacionando idade e comprimento total.

\section{MATERIAL E MÉTODOS}

O presente trabalho foi realizado no Laboratório de Tilapicultura do Centro de Aquicultura da Universidade Estadual Paulista e no biotério do Departamento de Morfologia e Fisiologia Animal (DMFA) do Campus de Jaboticabal, por um período de 60 dias.

As análises histológicas de gônadas e de microscopia eletrônica de varredura da boca foram processadas no Laboratório de Histologia de Peixes do DMFA e no Laboratório de Microscopia Eletrônica da Faculdade de Ciências 
Agrárias e Veterinárias da UNESP, Campus de Jaboticabal, respectivamente.

Para estudo do desenvolvimento do aparato bucal, foram utilizadas larvas provenientes de reprodutores de tilápias-do-nilo (Oreochromis niloticus) acondicionados em tanques de alvenaria com fluxo de água contínuo. Em cada aquário experimental, foi colocado um macho e três fêmeas por metro cúbico de água, num total de seis tanques com $2 \mathrm{~m}^{3}$ de capacidade cada um. Após a desova natural, os ovos foram retirados da boca de cada fêmea e incubados artificialmente para a observação do momento exato da eclosão das larvas. No quinto dia póseclosão, quinze larvas foram coletadas em intervalos de quatro em quatro horas. Para a determinação do tamanho da boca, as larvas foram fixadas em solução de Karnovsky (paraformaldeído + glutaraldeído) por 24 horas, lavadas em tampão cacodilato de sódio $0,1 \mathrm{M}$ e $\mathrm{pH} 7,4$, pós-fixadas em tetróxido de ósmio a $1 \%$ por 2 horas, lavadas novamente no mesmo tampão, desidratadas em série crescente de etanol, secas em secadora de ponto crítico com $\mathrm{CO}_{2}$ líquido, montadas em suporte de cobre com a boca voltada para a superfície e outras em decúbito lateral, metalizadas com ouro paládio, observadas e eletronmicrografadas em microscópio eletrônico de varredura ${ }^{1}$.

A medida da boca das larvas, em micrômetros $(\mu \mathrm{m})$, foi realizada em programa próprio do microscópio eletrônico de varredura ${ }^{1}$ (Fig. 1).

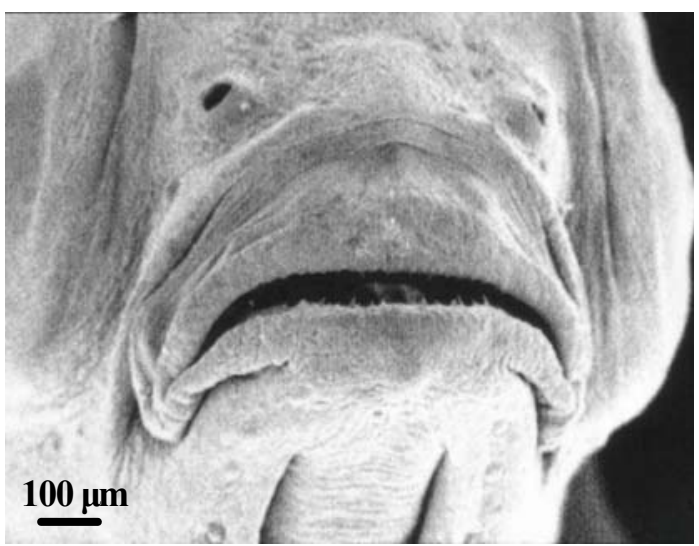

Figura1. Eletronmicrografia do aparato bucal de larva de tilápia-do-nilo com cinco dias póseclosão.

${ }^{1}$ Jeol - JSM 5410 - Tóquio, Japão.
Para testar as granulometrias de ração, utilizaram-se larvas de cinco dias de idade com peso médio de $0,0235 \mathrm{~g}$ e comprimento inicial de $10,57 \mathrm{~mm}$ que foram colocadas em caixas plásticas de 130L de capacidade e 100L de volume útil, com fluxo de água e aeração contínua. Foi realizado o monitoramento inicial dos parâmetros físico-químicos que caracterizaram a qualidade da água: temperatura, $\mathrm{pH}$, oxigênio, alcalinidade, dureza e amônia.

A ração contendo $35 \%$ de proteína bruta, 5,2\% de fibra bruta, $6,2 \%$ de extrato etéreo e $4130 \mathrm{Kcal}$ de energia bruta $/ \mathrm{kg}$, recebeu o hormônio masculinizante 17- $\alpha$-metiltestosterona, na concentração de $60 \mathrm{mg} / \mathrm{kg}$, e foi fornecida às larvas por 30 dias, nas granulometrias especificadas. Após o período de reversão, os animais receberam ração de crescimento na granulometria $0,35 \mathrm{~mm}$ até 60 dias. Foram colocadas, em cada caixa, 100 larvas, sendo que cada tratamento (três tratamentos) teve três repetições, totalizando nove caixas. Estas foram sifonadas todos os dias para remoção de fezes e ração não ingerida, a fim de manter a qualidade da água.

Com 30 e 60 dias de arraçoamento, foram amostrados 10 peixes de cada repetição para análises biométricas. Neste período, foram contados os peixes em cada caixa para o cálculo da taxa de sobrevivência. A sobrevivência foi calculada utilizando-se a fórmula: $\mathrm{TS}=$ número de peixes vivos/número total de peixes x 100 . Para análise histológica de gônadas, foram amostrados 10 peixes aos 35 dias de idade.

Para a biometria, os peixes foram fixados em solução de formol a $10 \%$ por 24 horas. Em seguida, foram lavados em água corrente, colocados em placas de Petri, pesados em balança analítica e medidos com paquímetro digital. As análises estatísticas foram feitas por meio do programa estatístico SAS/2004.

A retirada das gônadas foi feita manualmente, dissecadas e separadas por tratamento e por repetição. Foram então fixadas em formol tamponado $10 \%$, posteriormente desidratadas em série crescentes de etanol, diafanizadas em xilol, incluídas em parafina, seccionadas no micrótomo (5 $\mu \mathrm{m}$ de espessura) e as lâminas coradas pela técnica da hematoxilina-eosina. A fotodocumentação das lâminas foi realizada em 
fotomicroscópio ${ }^{2}$, acoplado a câmera de vídeo digital $^{3}$.

\section{RESULTADOS E DISCUSSÃO}

A média de temperatura da água nas caixas variou de $25,9 \pm 1,36^{\circ} \mathrm{C}$ a $28,0 \pm 0,62^{\circ} \mathrm{C}$, e foi aferida todos os dias às nove horas da manhã para permanecer dentro dos parâmetros ideais de acordo com Vinatea e Vega (1995). Os parâmetros físico-químicos tomados apresentaram os seguintes valores: $\mathrm{pH} 7,7 \pm 0,1$, oxigênio $\quad 8,8 \pm 0,5 \mathrm{mg} / \mathrm{L}, \quad$ alcalinidade $146 \pm 11 \mathrm{mg} / \mathrm{L}$, dureza $34 \pm 5 \mathrm{mg} / \mathrm{L}$ e amônia $0,12 \pm 0,1 \mathrm{mg} / \mathrm{L}$, estando dentro dos valores preconizados para a aquicultura e para o cultivo da espécie (Tachibana et al., 2004).

De acordo com Lovshin (1997), evidências sugerem que tilápias adultas utilizam rações fareladas tão eficientemente quanto as peletizadas, em tanques, quando estocadas de um a dois indivíduos por $\mathrm{m}^{2}$. No entanto, para as larvas, o problema aumenta tendo em vista que nessa fase os indivíduos são pequenos, necessitando de uma ração com tamanho de partícula adequado ao de sua boca (Kubitza, 1997).

As medidas da boca apresentaram valores médios de $918,2 \pm 152,9 \mu \mathrm{m}$ aos cinco dias de idade, tendo um tamanho compatível a todas as granulometrias testadas.

Meurer et al. (2005) observaram que para tilápias-do-nilo, com início de alimentação aos três dias de idade, a granulometria de $0,50 \mathrm{~mm}$ resultou na maior eficiência teórica para ganho de peso, comprimento e sobrevivência. Lovell (1988) e Kubitza (1997) sugeriram que o grau de moagem dos alimentos deve ser menor que $0,50 \mathrm{~mm}$. Esses autores referem-se à piscicultura de modo geral, porém, deve-se considerar a espécie de peixe e sua fase de criação. De acordo com Bombardelli et al. (2004), o fornecimento de ração farelada apresenta excelentes resultados produtivos para tilápia-do-nilo e ainda é de processamento mais simples e barato se comparada com rações micropeletizadas.

Os valores de biometria e sobrevivência são apresentados nas Tab. 1 e 2.

Tabela 1. Estatística $\mathrm{F}$ e coeficiente de variação obtido na análise da variância para comprimento total $(\mathrm{mm})$ e peso $(\mathrm{g})$ para a tilápia-do-nilo

\begin{tabular}{lcc}
\multicolumn{1}{c}{ Estatística } & Comprimento total $(\mathrm{mm})$ & Peso $(\mathrm{g})$ \\
\hline F para idade & $57,59^{*}$ & $94,81^{*}$ \\
F para tratamento & $0,55^{* *}$ & $1,97^{* *}$ \\
F para interação idade x tratamento & $0,48^{* *}$ & $1,80^{* *}$ \\
Coeficiente de variação & $11,61 \%$ & $25,44 \%$ \\
\hline
\end{tabular}

*significativo $(\mathrm{P}<0,05)$; **não significativo $(\mathrm{P}>0,05)$.

Tabela 2. Valores médios obtidos para sobrevivência (\%), peso (g) e comprimento total (mm), por idade e tratamento para a tilápia-do-nilo

\begin{tabular}{ccccc}
\hline Variável & Idade & \multicolumn{3}{c}{ Tratamento } \\
\cline { 3 - 5 } & & $0,25 \mathrm{~mm}$ & $0,35 \mathrm{~mm}$ & $0,50 \mathrm{~mm}$ \\
\hline Sobrevivência & 30 dias & $84,00 \mathrm{~A}$ & $86,00 \mathrm{~A}$ & $77,66 \mathrm{~A}$ \\
$(\%)$ & 60 dias & $27,00 \mathrm{~B}$ & $22,50 \mathrm{~B}$ & $25,33 \mathrm{~B}$ \\
\hline Peso & 30 dias & $0,53 \mathrm{~A}$ & $0,49 \mathrm{~A}$ & $0,50 \mathrm{~A}$ \\
$(\mathrm{~g})$ & 60 dias & $4,43 \mathrm{~B}$ & $2,92 \mathrm{~B}$ & $3,07 \mathrm{~B}$ \\
\hline Comprimento Total & 30 dias & $29,56 \mathrm{~A}$ & $29,23 \mathrm{~A}$ & $29,32 \mathrm{~A}$ \\
$(\mathrm{~mm})$ & 60 dias & $55,60 \mathrm{~B}$ & $48,07 \mathrm{~B}$ & $52,84 \mathrm{~B}$ \\
\hline
\end{tabular}

Médias seguidas por letras distintas na coluna diferem entre si pelo teste Tukey $(\mathrm{P}<0,05)$; não houve diferença entre os tratamentos $(\mathrm{P}>0,05)$.

\footnotetext{
${ }^{2}$ Leica DM 2500 - Alemanha.

${ }^{3}$ Leica DFC 280 - Alemanha.
} 
Não houve diferença entre tratamentos quando se compararam os valores médios de peso e comprimento total tomados aos 30 dias (Tab. 2). Makino (2005) também não encontrou diferenças estatísticas para comprimento total e ganho de peso em juvenis alimentados com rações de 0,25 ; 0,35 e $0,50 \mathrm{~mm}$. Produtores de tilápias nilóticas podem fazer uso de rações com qualquer uma das três granulometrias propostas sem prejuízo ao desempenho e à sobrevivência para larvas com início da alimentação aos cinco dias de idade. Porém, recomenda-se o uso da ração de $0,50 \mathrm{~mm}$ por ser de baixo custo em relação às demais.

Segundo Hayashi et al. (1999), ao avaliarem diferentes graus de moagem de ração para tilápias-do-nilo, verificaram que a granulometria de $0,50 \mathrm{~mm}$ apresentou melhores resultados de desempenho zootécnico. Soares et al. (2003) recomendaram o uso de ração peletizada com granulometria de $0,79 \mathrm{~mm}$ para tilápias nilóticas em fase de crescimento.

Quanto à biometria realizada com 30 e 60 dias de idades, houve diferenças significativas entre elas, o que já era esperado, pois com o avanço da idade naturalmente há crescimento e aumento de peso nos peixes, em condições ideais de nutrição, manejo e sanidade. Quanto à queda da sobrevivência aos 60 dias, esta pode ser atribuída à alta densidade com o crescimento dos peixes, tendo-se constatado que alguns estavam mortos, sem cauda ou machucados.

Microscopicamente, aos 35 dias, a maioria $(88,7 \%)$ das gônadas encontrava-se indiferenciadas (Fig. 2A) e as poucas restantes estavam revertidas em machos (Fig. 2B). O mesmo foi descrito por Makino et al. (2009), ao observar que a sexagem histológica em tilápias nilóticas aos 35 dias de idade mostravam gônadas imaturas e com grande quantidade de tecido de natureza conjuntivo-fibrosa. Aos 35 dias de idade, os peixes são ainda muito pequenos, tornando difícil a retirada e o processamento histológico das gônadas. Recomenda-se que a análise da sexagem histológica deva ser realizada de acordo com o tamanho dos animais e não de acordo com a idade.

Hiott e Phelps (1993) estudaram o tamanho e a idade das larvas de tilápias nilóticas na reversão sexual e constataram que o tamanho das larvas foi mais importante que a idade para o sucesso da reversão sexual. Carvalho e Foresti (1996) também analisaram a estrutura histológica das gônadas de tilápias com alimentação iniciada com menos de uma semana pós-eclosão e observaram que, em indivíduos que não receberam hormônio masculinizante, a diferenciação total das gônadas dá-se aos 48 dias nas fêmeas e 68 dias nos machos.

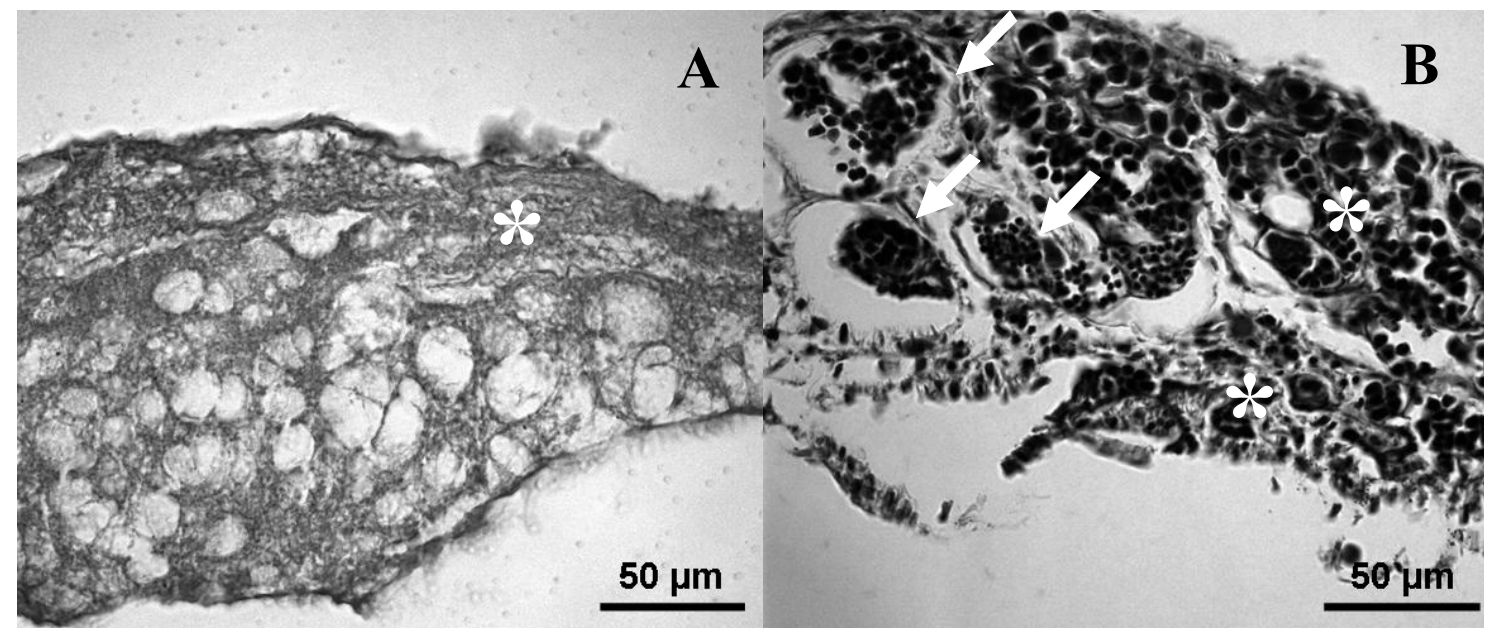

Figura 2. Fotomicrografias de gônadas de tilápia-do-nilo aos 35 dias de idade. A: Predominância de tecido conjuntivo com células indiferenciadas (40x - HE); B: Gônada com início de reversão sexual para macho (40x - HE). Setas: lóbulos seminíferos; asterisco: tecido conjuntivo com células indiferenciadas. 


\section{CONCLUSÕES}

A utilização de rações com granulometrias de $0,25 \mathrm{~mm}, 0,35 \mathrm{~mm}$ e $0,50 \mathrm{~mm}$ pode ser feita sem prejuízo ao desempenho e à sobrevivência para larvas de tilápia-do-nilo com início da alimentação aos cinco dias de idade. A análise de gônadas após a reversão não é indicada aos 35 dias de idade.

\section{REFERÊNCIAS BIBLIOGRÁFICAS}

BOMBARDELLI, R.A.; HAYASHI, C.; MEURER, F. et al. Avaliação de rações fareladas e micropeletizadas para larvas de tilápia do Nilo (Oreochromis niloticus) - desempenho e efetividade da reversão sexual. Acta Sci. Anim. Sci., v.26, p.197-201, 2004.

BOOTH, M.A.; ALLAN, G.L.; WARNERSMITH, L. Effects of grinding, steam conditioning and extrusion of practical diet on digestibility and weight gain of silver perch, Bidtanus bydianus. Aquaculture, v.182, p.287299, 2000

CARRASCO, L.A.P.; PENMAN, D.J.; VILLALOBOS, S.A. et al. The effects of oral administration with $17 \alpha$ - methyltestosterone on chromossomal synapses in Oreochromis niloticus (Pisces, Cichlidae). Mutat. Res., v.430, p.87-98, 1999.

CARVALHO, E.D.; FORESTI, F. Reversão de sexo em tilápia-do-nilo Oreochromis niloticus induzida por 17- $\alpha$-metiltestosterona: proporção de sexo e histologia das gônadas. Braz. J. Biol., v.56, p.249-262, 1996.

GALMAN, O.R.; AVTALION, R.R. Further study of the embryonic development of the Oreochromis niloticus (Ciclidae, teleostei) using scanning electron microscopy. J. Fish Biol., v.34, p.653-664, 1989.

HAYASHI, C.; BOSCOLO, W.R.; SOARES, C.M. et al. Uso de diferentes graus de moagem dos ingredientes em dietas para tilápia do Nilo (Oreochromis niloticus) na fase de crescimento. Acta Sci. Anim. Sci., v.21, p.733 - 737, 1999.

HIOTT, A.E.; PHELPS, R.P. Effects of initial age and size on Sex reversal of Oreochromis niloticus fry using methyltestosterone. Aquaculture, v.112, p.301-308, 1993.
KUBITZA, F. Nutrição e alimentação dos peixes. Piracicaba: ESALQ, 1997. 74p.

LOVELL, T. Nutrition and feeding of fish. New York: Van Nostrand and Reinhold, 1988. 284p.

LOVSHIN, L.L. Tilápia farming a growing worldwild aquaculture industry. In: SIMPOSIO SOBRE MANEJO E NUTRIÇÃO DE PEIXES, 1., 1997. Piracicaba, SP. Anais... Piracicaba: Colégio Brasileiro de Nutrição Animal, 1997. p.137. (Resumo).

MAINARDES-PINTO, C.S.R.; FENERICHVERANI, N.; CAMPOS, B.E.S. et al. Masculinização da Tilápia do Nilo, Oreochromis niloticus, utilizando diferentes rações e diferentes doses de $17 \alpha$-metiltestosterona. Rev. Bras. Zootec., v.29, p.654-659, 2000.

MAKINO, L.C. Validação dos métodos de identificação do sexo em tilápias-do-nilo (Oreochromis niloticus) revertidas com rações contendo diferentes granulometrias e de diferentes idades. 2005. 31f. Dissertação (Mestrado) - Centro de Aquicultura da Universidade Estadual Paulista, Jaboticabal.

MAKINO, L.C.; NAKAGHI, L.S.O.; PAES, M.C.F. et al. Efetividade de métodos de identificação sexual em tilápias-do-nilo (Oreochromis niloticus) revertidas sexualmente com hormônio em ração com diferentes granulometrias. Biosc. J., v.25, p.112-121, 2009.

MEURER, F.; HAYASHI, C.; BOSCOLO; W.R. et al. Influência do processamento da ração no desempenho e sobrevivência da tilápia do Nilo durante a reversão sexual. Rev. Bras. Zootec., v.32, p.262-267, 2003.

MEURER, F.; BOMBARDELLI, R.A.; HAYASHI, C. Grau de moagem dos alimentos em rações para a tilápia do Nilo (Oreochromis niloticus) durante o período de reversão sexual. Acta Sci. Anim. Sci., v.27, p.81-85, 2005.

MORRISON, C.M.; MIYAKE, T.; WRIGHT Jr, J.R. Histological study of the development of the embryo and early larva of Oreochromis niloticus (Pisces: Cichlidae). J. Morphol., v.247, p.172195, 2001.

NUTRIENT requirement of fish. Washington: National Academy of Sciences, 1993. 128p.

PEZZATO, L.E. Alimentação de peixes Relação custo benefício. In: REUNIÃO ANUAL DA SOCIEDADE BRASILEIRA DE 
ZOOTECNIA, 36., 1999, Porto Alegre. Anais.... Porto Alegre: SBZ, 1999. p.109. (Resumo).

PHELPS, R.P.; SLAZAR, G.C; ABE, V. Sex reversal and nursery growth of Nile tilapia, (Oreochromis niloticus, L.), free - swimming in earthen ponds. Aquac. Res., n. 26, p.293-295, 1995.

PINHEIRO, L.M.S.; MARTINS, R.T.; PINHEIRO, L.A.S. et al. Rendimento industrial de filetagem da tilápia tailandesa (Oreochromis spp.). Arq. Bras. Med. Vet. Zootec., v.58, p.257262, 2006.

SANTIAGO, C.B.; ALDABA, M.B.; REYES, O.S. Influence of feeding rate and diet from on growth and survival of Nile tilapia (Oreochromis niloticus) fry. Aquaculture, v.64, p.277-282, 1987.
SOARES, C.M.; HAYASHI, C.; BOSCOLO, W.R. et al. Diferentes graus de moagem dos ingredientes em dietas peletizadas para a tilápia do Nilo (Oreochromis niloticus L.) em fase de crescimento. Desempenho e digestibilidade aparente. Zootec. Trop., v.21, p.275-287, 2003.

TACHIBANA, L.; CASTAGNOLLI, N.; PEZZATO, L.E. et al. Desempenho de diferentes linhagens de tilápia do Nilo (Oreochromis niloticus) na fase de reversão sexual. Acta Sci. Anim. Sci., v.26, p.305-311, 2004.

TOYAMA, G.N.; CORRENTE, J.E.; CYRINO, J.E.P. Suplementação de vitamina $\mathrm{C}$ em rações para reversão sexual da tilápia do Nilo. Sci. Agric., v.57, p.221-228, 2000.

VINATEA, J.E.; VEGA, A.L. Piscicultura tropical: especies nativas y exoticas. Lima: Copyright, 1995. 338p. 